\title{
Review: pharmacological and non-pharmacological interventions improve outcomes in patients with dementia and in their caregivers
}

\author{
Doody RS, Stevens JC, Beck C, et al. Practice parameter: management of dementia (an evidence-based review). Report of the \\ Quality Standards Subcommittee of the American Academy of Neurology. Neurology 2001 May 8;56:1154-66.
}

\section{QUESTIONS: Do pharmacotherapy, educational, or other non-pharmacological} interventions improve outcomes in patients with dementia or in their caregivers?

\section{Data sources}

Studies were identified by searching Medline, EMBASE/ Excerpta Medica, CINAHL, Current Contents, Psych Abstracts, PsycInfo, and the Cochrane databases using search terms including Alzheimer's disease (AD), vascular or multi infarct dementia, dementia with associated parkinsonian disorder, progressive supranuclear palsy, frontotemporal dementia, and senile dementia. Additional search terms used were question specific. Bibliographies of relevant papers were also reviewed.

\section{Study selection}

Studies were selected if they were randomised controlled trials published in any language or other types of studies published in English with $>20$ participants.

\section{Data extraction}

Data were extracted on study quality, participant characteristics, interventions, outcome measures, and results.

\section{Main results}

380 papers met the selection criteria. Several studies comparing cholinesterase inhibitors (eg, tacrine, donepezil, tartrate, and galantamine) with placebo showed that the drugs were more effective than placebo for improving cognitive outcomes in a subgroup of patients with mild to moderate AD. Studies of cholinergic precursors (eg, lecithin) and muscarinic agonists (eg, xanomeline) for the treatment of $\mathrm{AD}$ have not shown beneficial effects. 1 large 2 year study showed that selegiline, $5 \mathrm{mg}$ taken orally twice daily, and vitamin $\mathrm{E}$, $1000 \mathrm{IU}$ taken orally twice daily, delayed the time to a composite outcome indicator of clinical worsening of $\mathrm{AD}$ symptoms; however, no additive effects were seen from the combined use of selegiline and vitamin E. Data are lacking to support the use of other antioxidant, antiinflammatory, or other putative disease modifying drugs in the treatment of AD. Antipsychotic drugs were effective for treating agitation or psychosis in patients with dementia where environmental manipulation failed, and antidepressants (eg, selected tricyclics, monoamine oxidase B inhibitors, and selective serotonin reuptake inhibitors) were effective for treating depression in patients with dementia. Evidence from observational studies showed that educating family caregivers of patients with $\mathrm{AD}$ improved caregiver satisfaction and delayed time to patient's institutionalisation and that educating staff in long term care facilities about $\mathrm{AD}$ minimised the unnecessary use of antipsychotic drugs. Randomised trials have shown that behaviour modification, scheduled toileting, and prompted voiding reduced urinary incontinence in people with dementia. Graded assistance, skills practice, and positive reinforcement increased functional independence.

\section{Conclusions}

Cholinesterase inhibitors improve outcomes in some patients with Alzheimer's disease (AD). Antipsychotics may be effective for treating agitation and antidepressants for treating depression in patients with dementia. Education for family caregivers of patients with $\mathrm{AD}$ may improve caregiver and patient outcomes. Nonpharmacological interventions such as behavioural modification are also effective.
Source of funding:
no external funding.

For correspondence: Quality Standards Subcommittee,

American Academy of

Neurology, 1080

Montreal Avenue, St Paul, MN 55116, USA. Fax +1713798

5326.

A modified version of this abstract and commentary appears in ACP Journal Club.

A modified version of this abstract appears in Evidence-Based

Nursing.

\section{COMMENTARY}

Doody et al have used a rigorous methodology and broadly covered the literature on the management of dementia. This review addresses all of the relevant issues relating to treating the patient with dementia and offers sound advice. Although the authors suggest that the guideline is directed to neurologists and all other clinicians who manage dementia, the review may also be relevant to the primary care physician. Other excellent documents that do explicitly address the primary care physician have been published by a Canadian group. ${ }^{1,2}$

These guidelines by Doody et al provide an excellent review of the current literature of anti-dementia drugs, non-pharmacological interventions, and educational interventions. Furthermore, the authors offer sensible recommendations for future research.

Although Doody et al offer guidelines for the use of 3 main drugs (the cholinesterase inhibitors donepezil, rivastigmine, and galantamine), they point out that because no head to head trials of these drugs exist, they cannot recommend one over the other. Therefore, at present, the differences in dosing schedules and side effect profiles should be considered when choosing which drug to use.

For treatment of the patient with $\mathrm{AD}$, there are excellent studies on drug treatment and good papers on educational and non-pharmacological interventions. Educational and non-pharmacological interventions are probably under used by many, but may be as effective as drug treatment and do not present the burdens of side effects or substantial costs.

Although the Cochrane databases were consulted, none of the relevant reviews was cited (eg, the reviews on selegiline ${ }^{3}$ and thioridazine ${ }^{4}$ ). These guidelines, however, still provide an excellent summary of the issues on the treatment of dementia from a very broad perspective.

A Mark Clarfield, MD, FRCPC Soroka Hospital Centre and Ben Gurion University of the Negev Jerusalem, Israel

1 Clarfield AM, Bass MJ, Cohen C, et al. Assessing dementia: the Canadian Consensus (editorial). Can Med Assoc J 1991;144:851-3.

2 Patterson CJ, Gauthier S, Bergman H, et al. The recognition, assessment and management of dementing disorders: conclusions from the Canadian Consensus Conference on Dementia. Can Med Assoc J 1999;160(12 Suppl):S1-15.

3 Birks J, Flicker L. Selegiline for Alzheimer's disease. Cochrane Database Syst Rev 2001;(2):CD000442.

4 Kirchner V, Kelly CA, Harvey RJ. Thioridazine for dementia. Cochrane Database Syst Rev 2001;(2):CD000464. 